$\mathrm{ROM} 2 \mathrm{~F} / 98 / 11$

\title{
Gravitational Wave Radiation from Compact Binary Systems in the Jordan-Brans-Dicke Theory
}

\author{
M. Brunetti甲, E. Coccia ${ }^{12}$, V. Fafone
}

\begin{abstract}
In this paper we analyze the signal emitted by a compact binary system in the Jordan-Brans-Dicke theory. We compute the scalar and tensor components of the power radiated by the source and study the scalar waveform. Eventually we consider the detectability of the scalar component of the radiation by interferometers and resonant-mass detectors.
\end{abstract}

\footnotetext{
${ }^{1}$ Dipartimento di Fisica, Università di Roma II "Tor Vergata"

${ }^{2}$ I.N.F.N. Sezione di Roma II, Via Della Ricerca Scientifica, 00133 Roma, ITALY

${ }^{3}$ I.N.F.N. Laboratori Nazionali di Frascati, Via E. Fermi 40, 00044 Frascati (Roma), ITALY
} 


\section{Introduction}

The detection of gravitational waves $(\mathrm{GW})$ is a field of active research from the point of view both of the development of suitable detectors and of the study of possible sources and signal analysis. The detectors now operating as GW observatories are of the resonant-mass type and have a sensitivity to typical millisecond GW bursts of $h \approx 6 \times 10^{-19}$ ( $h$ is the wave amplitude) or, in spectral units, $10^{-21}(\mathrm{~Hz})^{-1 / 2}$ over a bandwidth of a few $\mathrm{Hz}$ around $1 \mathrm{kHz}$ [1]. The first bound is appropriate for describing the sensitivity to gravitational collapses while the square of the second bound represents the input GW spectrum that would produce a signal equal to the noise spectrum actually observed at the output of the detector. With this sensitivity it is possible to monitor the strongest potential sources of GW in our galaxy and in the local group (distances of $\approx 1 \mathrm{Mpc}$ ). In order to improve the sensitivity of these instruments, more advanced transducers and amplifiers are under development as well as new resonant-mass detectors of spherical shape. Furthermore a huge effort is under way to build large laser interferometers. It is widely believed that in the near future, sensitivities of the order of $10^{-23}(\mathrm{~Hz})^{-1 / 2}$ over a bandwidth of several hundred $\mathrm{Hz}$ will be attained allowing the observation of GW sources up to distances of the order of $100 \mathrm{Mpc}$ [1]. It thus seems that the detection of GWs is highly probable at the beginning of the new millennium. In addition to information of astrophysical interest, the detection of GW gives an opportunity to test the content of the theory of gravity. In fact, it has been shown that a single spherical resonantmass detector [2], or an array of interferometers [3], have the capability to probe the spin content of the incoming GW.

One of the most intensively studied GW source is the inspiralling compact binary system [4] made of neutron stars or black holes. In the Newtonian regime, the system has a clean analytic behaviour and emits a wave-form of increasing amplitude and frequency that can sweep up to the $\mathrm{kHz}$ range of frequencies. In this paper we 
study the radiation emitted by this source in the framework of the Jordan-BransDicke (JBD) theory. We consider this theory to be of particular interest, since the coupling between the scalar field and the metric has the same form of that of string theory, which is widely believed to give a consistent quantum extension of classical gravity. Our main motivation then comes from the attemp to explore a possible experimental signature of string theory as already discussed in [2]. Furthermore the results obtained here generalize to any theory with a JBD type coupling between matter and gravitation.

There has been much work in this domain in the past years. Before going to the plan of the paper, we shortly review it. In [5] binary systems were first proposed as possible sources from which extract more stringent bounds on $\omega_{B D}$ (see (2.1) for its definition) than those obtained from solar system data. An analysys of spherically symmetric collapse of inhomogeneous dust was carried on in [3] and later confirmed in [6]. The case of homogeneous dust was treated in [7]. In [8] a test particle around a Kerr black hole was studied and results very similar to those of our section 5.1 for interferometers were found. In [9] it was pointed out that deviations from general relativity can be much different in strong and weak gravity. In [10] these deviations were parametrized in a two dimensional space and exclusion plots were drawn out of the available data. Finally in [11] spherical collapses were studied in a formalism which kept in account strong gravity effects.

The plan of the paper is the following: in Section two we describe the scalar and tensor GW solutions of the JBD theory. In Section three we compute the power emitted in tensor and scalar GW by a binary system. In Section four we concentrate on the scalar waveform. In Section five, we study the interaction between the scalar waveform and two types of earth-based detectors: interferometers and spherical resonant-mass detectors, giving limits for the detectability of the signals coming from typical binary sources. Eventually, in Section six, we draw some conclusions. 


\section{Scalar and Tensor GWs in the JBD Theory}

In the Jordan-Fierz frame, in which the scalar field mixes with the metric but decouples from matter, the action reads 12

$$
\begin{aligned}
S & =S_{\text {grav }}\left[\phi, g_{\mu \nu}\right]+S_{\mathrm{m}}\left[\psi_{m}, g_{\mu \nu}\right] \\
& =\frac{c^{3}}{16 \pi} \int d^{4} x \sqrt{-g}\left[\phi R-\frac{\omega_{B D}}{\phi} g^{\mu \nu} \partial_{\mu} \phi \partial_{\nu} \phi\right]+\frac{1}{c} \int d^{4} x L_{\mathrm{m}}\left[\psi_{\mathrm{m}}, g_{\mu \nu}\right]
\end{aligned}
$$

where $\omega_{B D}$ is a dimensionless constant, whose lower bound is fixed to be $\omega_{B D} \approx 600$ by experimental data [13], $g_{\mu \nu}$ is the metric tensor, $\phi$ is a scalar field, and $\psi_{\mathrm{m}}$ collectively denotes the matter fields of the theory.

As a preliminary analysis, we perform a weak field approximation around the background given by a Minkowskian metric and a constant expectation value for the scalar field

$$
\begin{aligned}
g_{\mu \nu} & =\eta_{\mu \nu}+h_{\mu \nu} \\
\varphi & =\varphi_{0}+\xi .
\end{aligned}
$$

The standard parametrization $\varphi_{0}=2\left(\omega_{B D}+2\right) / G\left(2 \omega_{B D}+3\right)$, with $G$ the Newton constant, reproduces GR in the limit $\omega_{B D} \rightarrow \infty$, which implies $\varphi_{0} \rightarrow 1 / G$. Defining the new field

$$
\theta_{\mu \nu}=h_{\mu \nu}-\frac{1}{2} \eta_{\mu \nu} h-\eta_{\mu \nu} \frac{\xi}{\varphi_{0}}
$$

where $h$ is the trace of the fluctuation $h_{\mu \nu}$, and choosing the gauge

$$
\partial_{\mu} \theta^{\mu \nu}=0
$$

one can write the field equations in the following form

$$
\begin{aligned}
\partial_{\alpha} \partial^{\alpha} \theta_{\mu \nu} & =-\frac{16 \pi}{\varphi_{0}} \tau_{\mu \nu} \\
\partial_{\alpha} \partial^{\alpha} \xi & =\frac{8 \pi}{2 \omega_{B D}+3} S
\end{aligned}
$$


where

$$
\begin{aligned}
\tau_{\mu \nu} & =\frac{1}{\varphi_{0}}\left(T_{\mu \nu}+t_{\mu \nu}\right) \\
S & =-\frac{T}{2\left(2 \omega_{B D}+3\right)}\left(1-\frac{1}{2} \theta-2 \frac{\xi}{\varphi_{0}}\right)-\frac{1}{16 \pi}\left[\frac{1}{2} \partial_{\alpha}\left(\theta \partial^{\alpha} \xi\right)+\frac{2}{\varphi_{0}} \partial_{a}\left(\xi \partial^{\alpha} \xi\right)\right]
\end{aligned}
$$

In the equation (2.7), $T_{\mu \nu}$ is the matter stress-energy tensor and $t_{\mu \nu}$ is the gravitational stress-energy pseudo-tensor, that is a function of quadratic order in the weak gravitational fields $\theta_{\mu \nu}$ and $\xi$. The reason why we have written the field equations at the quadratic order in $\theta_{\mu \nu}$ and $\xi$ is that in this way, as we will see later, the expressions for $\theta_{\mu \nu}$ and $\xi$ include all the terms of order $(v / c)^{2}$, where $v$ is the typical velocity of the source (Newtonian approximation).

Let us now compute $\tau^{00}$ and $S$ at the order $(v / c)^{2}$. Introducing the Newtonian potential $U$ produced by the rest-mass density $\rho$

$$
U(\vec{x}, t)=\int \frac{\rho\left(\vec{x}^{\prime}, t\right)}{\left|\vec{x}-\vec{x}^{\prime}\right|} d^{3} x^{\prime}
$$

the total pressure $p$ and the specific energy density $\Pi$ (that is the ratio of energy density to rest-mass density) we get (for a more detailed derivation, see [14]):

$$
\begin{aligned}
\tau^{00} & =\frac{1}{\varphi_{0}} \rho \\
S & \simeq-\frac{T}{2\left(2 \omega_{B D}+3\right)}\left(1-\frac{1}{2} \theta-2 \frac{\xi}{\varphi_{0}}\right) \\
& =\frac{\rho}{2\left(2 \omega_{B D}+3\right.}\left(1+\Pi-3 \frac{p}{\rho}+\frac{2 \omega_{B D}+1}{\omega_{B D}+2} U\right)
\end{aligned}
$$

Far from the source, the equations (2.5) and (2.6) admit wave-like solutions, which are superpositions of terms of the form

$$
\begin{aligned}
\theta_{\mu \nu}(x) & =A_{\mu \nu}(\vec{x}, \omega) \exp \left(i k^{\alpha} x_{\alpha}\right)+c . c . \\
\xi(x) & =B(\vec{x}, \omega) \exp \left(i k^{\alpha} x_{\alpha}\right)+c . c .
\end{aligned}
$$

Without affecting the gauge condition (2.4), one can impose $h=-2 \xi / \varphi_{0}$ (so that $\left.\theta_{\mu \nu}=h_{\mu \nu}\right)$. Gauging away the superflous components, one can write the amplitude 
$A_{\mu \nu}$ in terms of the three degrees of freedom corrisponding to states with helicities \pm 2 and 0 [15]. For a wave travelling in the $z$-direction, one thus obtains

$$
A_{\mu \nu}=\left(\begin{array}{cccc}
0 & 0 & 0 & 0 \\
0 & e_{11}-b & e_{12} & 0 \\
0 & e_{12} & -e_{11}-b & 0 \\
0 & 0 & 0 & 0
\end{array}\right)
$$

where $b=B / \varphi_{0}$.

\section{Power emitted in GWs}

The power emitted by a source in GWs depends on the stress-energy pseudo-tensor $t^{\mu \nu}$ according to the following expression

$$
P_{G W}=r^{2} \int \Phi d \Omega=r^{2} \int<t^{0 k}>\hat{x}_{k} d \Omega
$$

where $r$ is the radius of a sphere which contains the source, $\Omega$ is the solid angle, $\Phi$ is the energy flux and the symbol $\langle\ldots\rangle$ implies an average over a region of size much larger than the wavelength of the GW. At the quadratic order in the weak fields we find

$$
<t_{0 z}>=-\hat{z} \frac{\varphi_{0} c^{4}}{32 \pi}\left[\frac{4\left(\omega_{B D}+1\right)}{\varphi_{0}^{2}}<\left(\partial_{0} \xi\right)\left(\partial_{0} \xi\right)>+<\left(\partial_{0} h_{\alpha \beta}\right)\left(\partial_{0} h^{\alpha \beta}\right)>\right] .
$$

Substituting (2.12), (2.13) into (3.2), one gets

$$
<t_{0 z}>=-\hat{z} \frac{\varphi_{0} c^{4} \omega^{2}}{16 \pi}\left[\frac{2\left(2 \omega_{B D}+3\right)}{\varphi_{0}^{2}}|B|^{2}+A^{\alpha \beta *} A_{\alpha \beta}-\frac{1}{2}\left|A_{\alpha}^{\alpha}\right|^{2}\right],
$$

and using (2.14)

$$
<t_{0 z}>=-\hat{z} \frac{\varphi_{0} c^{4} \omega^{2}}{8 \pi}\left[\left|e_{11}\right|^{2}+\left|e_{12}\right|^{2}+\left(2 \omega_{B D}+3\right)|b|^{2}\right] .
$$

From (3.4) we see that the purely scalar contribution, associated to $b$, and the traceless tensorial contribution, associated to $e_{\mu \nu}$, are completely decoupled and can thus be treated independently. 


\subsection{Power emitted in tensor GWs}

Eq. (2.7) differs from the corresponding tensor field in GR only by a multiplicative factor. Then we can directly write the final result using the well-known expression for the power emitted by a system of binary stars in GR

$$
\left(P_{t e n}\right)_{n}=\frac{1}{G \varphi_{0}}\left(P_{G R}\right)_{n}
$$

If we take $\omega_{B D}=600$ in (3.5), the multiplicative factor $1 / G \varphi_{0}$ differs from one for one part in $10^{3}$.

In (3.5) $\left(P_{G R}\right)_{n}$ is the power emitted at frequency $n \omega_{0}$ (where $\omega_{0}$ is the orbital frequency) by a system of binary stars according to GR [16, 17], averaged over one period of the elliptical motion and calculated in the Newtonian approximation

$$
\left(P_{G R}\right)_{n}=\frac{32}{5} \frac{G^{4}}{c^{5}} \frac{m_{1}^{2} m_{2}^{2} m}{a^{5}} g(n ; e)
$$

where $m_{1}$ and $m_{2}$ are the masses of the two stars, $m$ is the total mass $m=m_{1}+m_{2}$, $a$ is the major semi-axis and $e$ is the eccentrity of the ellipse. The function $g(n ; e)$ depends on the Bessel functions $J_{k}(n e)$

$$
\begin{aligned}
g(n ; e) & =\frac{n^{4}}{8}\left[J_{n}^{2}(n e)\left(e^{2}-2\right)^{2} /\left(n^{2} e^{4}\right)+4 J_{n}^{2}(n e)\left(1-e^{2}\right)^{3} / e^{4}\right. \\
& +J_{n}^{2}(n e) /\left(3 n^{2}\right)+4 J_{n}(n e) J_{n}^{\prime}(n e)\left(e^{2}-2\right)\left(1-e^{2}\right) /\left(n e^{3}\right) \\
& \left.-8 J_{n}(n e) J_{n}^{\prime}(n e)\left(1-e^{2}\right)^{2} /\left(n e^{3}\right)\right] \\
& +4 J_{n}^{\prime 2}(n e)\left(1-e^{2}\right)^{2} / e^{2}+4 J_{n}^{\prime 2}(n e)\left(1-e^{2}\right) /\left(n^{2} e^{2}\right)
\end{aligned}
$$

In the last phases of the binary system evolution, the orbit becomes more and more circular, because the bodies radiate the most at their closest approach [16]. In the case of null eccentricity $e=0$, the function $g(n ; e)$ reduces to a Kronecker delta, $g(n ; e=0)=\delta_{n 2}$, and the tensor GW frequency is twice the orbital frequency. Summing over all the harmonics $n$ [16], one obtains

$$
P_{\text {ten }}=\sum_{n=1}^{\infty}\left(P_{\text {ten }}\right)_{n}=\frac{32}{5} \frac{G^{3}}{\varphi_{0} c^{5}} \frac{m_{1}^{2} m_{2}^{2} m}{a^{5}} f(e)
$$


where

$$
f(e)=\frac{1}{\left(1-e^{2}\right)^{7 / 2}}\left(1+\frac{73}{24} e^{2}+\frac{37}{96} e^{4}\right)
$$

Eqs. (3.6) and (3.8) are obtained in the approximation of point-like masses (weak self-gravity). For compact binary systems like PSR $1913+16$, they can be used upon replacing the masses, $m_{1}, m_{2}$, by the Schwarzschild masses of the stars [18].

\subsection{Power emitted in scalar GWs}

We now rewrite the scalar wave solution (2.13) in the following way

$$
\xi(\vec{x}, t)=\xi(\vec{x}, \omega) e^{-i \omega t}+\text { c.c. }
$$

In vacuo, the spatial part of the previous solution (3.10) satisfies the Helmholtz equation

$$
\left(\nabla^{2}+\omega^{2}\right) \xi(\vec{x}, \omega)=0
$$

The solution of (3.11) can be written as

$$
\xi(\vec{x}, \omega)=\sum_{j m} X_{j m} h_{j}^{(1)}(\omega r) Y_{j m}(\theta, \varphi)
$$

where $h_{j}^{(1)}(x)$ are the spherical Hankel functions of the first kind, $r$ is the distance of the source from the observer, $Y_{j m}(\theta, \varphi)$ are the scalar spherical harmonics and the coefficients $X_{j m}$ give the amplitudes of the various multipoles which are present in the scalar radiation field. Solving the inhomogeneous wave equation (2.6), we find

$$
X_{j m}=16 \pi i \omega \int_{V} j_{l}\left(\omega r^{\prime}\right) Y_{l m}^{*}(\theta, \varphi) S(\vec{x}, \omega) d V
$$

where $j_{l}(x)$ are the spherical Bessel functions and $r^{\prime}$ is a radial coordinate which assumes its values in the volume $V$ occupied by the source.

Substituting (3.2) in (3.1), considering the expressions (3.10) and (3.12), and averaging over time, one finally obtains

$$
P_{s c a l}=\frac{\left(2 \omega_{B D}+3\right) c^{4}}{8 \pi \varphi_{0}} \sum_{j m}\left|X_{j m}\right|^{2}
$$


To compute the power radiated in scalar GWs, one has to determine the coefficients $X_{j m}$, defined in (3.13). The detailed calculations can be found in the appendix A, while here we only give the final results. Introducing the reduced mass of the binary system $\mu=m_{1} m_{2} / m$ and the gravitational self-energy for the body $a$ (with $a=1,2$ )

$$
\Omega_{a}=-\frac{1}{2} \int_{V_{a}} \frac{\rho(\vec{x}) \rho\left(\vec{x}^{\prime}\right)}{\left|\vec{x}-\vec{x}^{\prime}\right|} d^{3} x d^{3} x^{\prime}
$$

one can write the Fourier components with frequency $n \omega_{0}$ in the Newtonian approximation as (see (A.15), A.23), (A.31), (A.32))

$$
\left(X_{00}\right)_{n}=-\frac{16 \sqrt{2 \pi}}{3} \frac{i \omega_{0} \varphi_{0}}{\omega_{B D}+2} \frac{m \mu}{a} n J_{n}(n e)
$$




$$
\begin{aligned}
\left(X_{1 \pm 1}\right)_{n}= & -\sqrt{\frac{2 \pi}{3}} \frac{2 i \omega_{0}^{2} \varphi_{0}}{\omega_{B D}+2}\left(\frac{\Omega_{2}}{m_{2}}-\frac{\Omega_{1}}{m_{1}}\right) \mu a \\
& {\left[ \pm J_{n}^{\prime}(n e)-\frac{1}{e}\left(1-e^{2}\right)^{1 / 2} J_{n}(n e)\right] } \\
\left(X_{20}\right)_{n}= & \frac{2}{3} \sqrt{\frac{\pi}{5}} \frac{i \omega_{0}^{3} \varphi_{0}}{\omega_{B D}+2} \mu a^{2} n J_{n}(n e) \\
\left(X_{2 \pm 2}\right)_{n}= & \mp 2 \sqrt{\frac{\pi}{30}} \frac{i \omega_{0}^{3} \varphi_{0}}{\omega_{B D}+2} \mu a^{2} \\
& \frac{1}{n}\left\{\left(e^{2}-2\right) J_{n}(n e) /\left(n e^{2}\right)+2\left(1-e^{2}\right) J_{n}^{\prime}(n e) / e\right. \\
\mp & \left.2\left(1-e^{2}\right)^{1 / 2}\left[\left(1-e^{2}\right) J_{n}(n e) / e^{2}-J_{n}^{\prime}(n e) /(n e)\right]\right\}
\end{aligned}
$$

Substituting these expressions in (3.14), leads to the power radiated in scalar GWs in the $n$-th harmonic

$$
\left(P_{s c a l}\right)_{n}=P_{n}^{j=0}+P_{n}^{j=1}+P_{n}^{j=2}
$$

where the monopole, dipole and quadrupole terms are respectively

$$
\begin{aligned}
P_{n}^{j=0} & =\frac{64}{9\left(\omega_{B D}+2\right)} \frac{m^{3} \mu^{2} G^{4}}{a^{5} c^{5}} n^{2} J_{n}^{2}(n e) \\
& =\frac{64}{9\left(\omega_{B D}+2\right)} \frac{m^{3} \mu^{2} G^{4}}{a^{5} c^{5}} m(n ; e) \\
P_{n}^{j=1} & =\frac{4}{3\left(\omega_{B D}+2\right)} \frac{m^{2} \mu^{2} G^{3}}{a^{4} c^{3}}\left(\frac{\Omega_{2}}{m_{2}}-\frac{\Omega_{1}}{m_{1}}\right)^{2} \\
& =\frac{4}{3\left(\omega_{B D}+2\right)} \frac{\left.m^{2}(n e)+\frac{1}{e^{2}}\left(1-e^{2}\right) J_{n}^{2}(n e)\right]}{a^{4} c^{3}}\left(\frac{\Omega_{2}}{m_{2}}-\frac{\Omega_{1}}{m_{1}}\right)^{2} d(n ; e) \\
P_{n}^{j=2} & =\frac{8}{15\left(\omega_{B D}+2\right)} \frac{m^{3} \mu^{2} G^{4}}{a^{5} c^{5}} g(n ; e)
\end{aligned}
$$

In figures $1,2,3$ we plot the monopole $m(n ; e)$, dipole $d(n ; e)$ and quadrupole $g(n ; e)$ functions against the index $n$, for different values of the eccentricity $e$. 


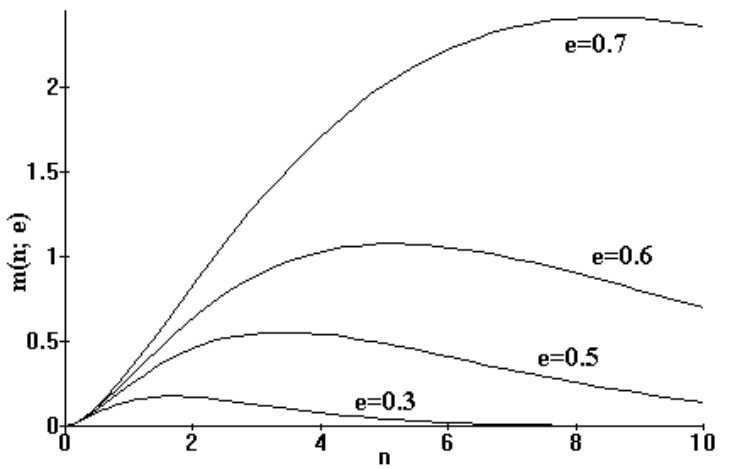

Figure 1

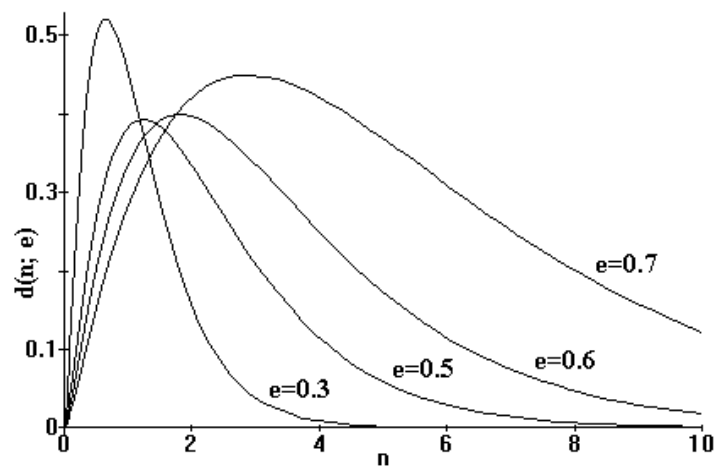

Figure 2

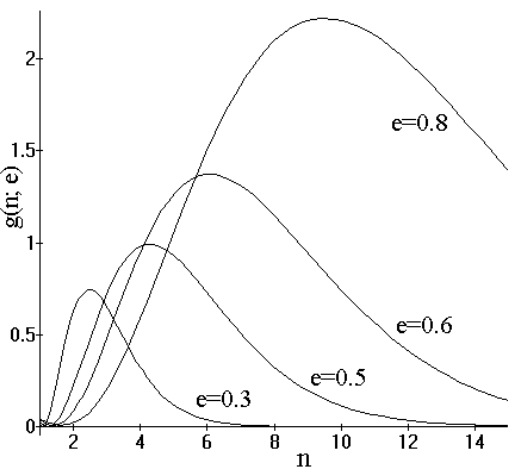

Figure 3 
From the figures one can infer the dominant harmonics in the scalar GWs radiation. In the case of circular orbit, the dipole function $d(n ; e)$ reduces to a Kronecker delta $d(n ; e=0)=\delta_{n 1}$, while the monopole function $m(n ; e)$ goes to zero.

The total power radiated in scalar GWs by a binary system is the sum of three terms

$$
P_{\text {scal }}=P^{j=0}+P^{j=1}+P^{j=2}
$$

where

$$
\begin{aligned}
P^{j=0} & =\frac{16}{9\left(\omega_{B D}+2\right)} \frac{G^{4}}{c^{5}} \frac{m_{1}^{2} m_{2}^{2} m}{a^{5}} \frac{e^{2}}{\left(1-e^{2}\right)^{7 / 2}}\left(1+\frac{e^{2}}{4}\right) \\
P^{j=1} & =\frac{2}{\omega_{B D}+2}\left(\frac{\Omega_{2}}{m_{2}}-\frac{\Omega_{1}}{m_{1}}\right)^{2} \frac{G^{3}}{c^{3}} \frac{m_{1}^{2} m_{2}^{2}}{a^{4}} \frac{1}{\left(1-e^{2}\right)^{5 / 2}}\left(1+\frac{e^{2}}{2}\right) \\
P^{j=2} & =\frac{8}{15\left(\omega_{B D}+2\right)} \frac{G^{4}}{c^{5}} \frac{m_{1}^{2} m_{2}^{2} m}{a^{5}} \frac{1}{\left(1-e^{2}\right)^{7 / 2}}\left(1+\frac{73}{24} e^{2}+\frac{37}{96} e^{4}\right)
\end{aligned}
$$

Note that $P^{j=0}, P^{j=1}, P^{j=2}$ all go to zero in the limit $\omega_{B D} \rightarrow \infty$.

\section{Scalar GWs}

We now give the explicit form of the scalar GWs radiated by a binary system. To this end, note that the major semi-axis, $a$, is related to the total energy, $E$, of the system through the following equation

$$
a=-\frac{G m_{1} m_{2}}{2 E}
$$

Let us consider the case of a circular orbit, remembering that in the last phase of evolution of a binary system this condition is usually satisfied. Furthermore we will also assume $m_{1}=m_{2}$. With these positions only the quadrupole term, (3.23), of the gravitational radiation is different from zero. The total power radiated in GWs, averaged over time, is then given by (3.8), (3.25)-(3.27)

$$
P=\frac{8}{15\left(\omega_{B D}+2\right)} \frac{G^{4}}{c^{5}} \frac{m_{1}^{2} m_{2}^{2} m}{d^{5}}\left[6\left(2 \omega_{B D}+3\right)+1\right]
$$


where $d$ is the relative distance between the two stars. The time variation of $d$ in one orbital period is

$$
\dot{d}=-\frac{G m_{1} m_{2}}{2 E^{2}} P
$$

Finally, substituting (4.1), (4.2) in (4.3) and integrating over time, one obtains

$$
d=2\left(\frac{2}{15} \frac{12 \omega_{B D}+19}{\omega_{B D}+2} \frac{G^{3} m_{1} m_{2} m}{c^{5}}\right)^{1 / 4} \tau^{4}
$$

where we have defined $\tau=t_{c}-t, t_{c}$ being the time of the collapse between the two bodies.

From (3.12), (3.16)-(3.19) and (B.3) one can deduce the form of the scalar field (see appendix B for details) which, for equal masses, is

$$
\xi(t)=-\frac{2 \mu}{r\left(2 \omega_{B D}+3\right)}\left[v^{2}+\frac{m}{d}-(\hat{n} \cdot \vec{v})^{2}+\frac{m}{d^{3}}(\hat{n} \cdot \vec{d})\right]
$$

where $r$ is the distance of the source from the observer, and $\hat{n}$ is the versor of the line of sight from the observer to the binary system center of mass. Indicating with $\gamma$ the inclination angle, that is the angle between the orbital plane and the reference plane (defined to be a plane perpendicular to the line of sight), and with $\psi$ the true anomaly, that is the angle between $d$ and the $x$-axis in the orbital plane $x$ - $y$, yields $\hat{n} \cdot \vec{d}=d \sin \gamma \sin \psi$. Then from (4.5) one obtains

$$
\xi(t)=\frac{2 G \mu m}{\left(2 \omega_{B D}+3\right) c^{4} d r} \sin ^{2} \gamma \cos (2 \psi(t))
$$

which can also be written as

$$
\xi(\tau)=\xi_{0}(\tau) \sin (\chi(\tau)+\bar{\chi})
$$

where $\bar{\chi}$ is an arbitrary phase and the amplitude $\xi_{0}(\tau)$ is given by

$$
\begin{aligned}
\xi_{0}(\tau) & =\frac{2 G \mu m}{\left(2 \omega_{B D}+3\right) c^{4} d r} \sin ^{2} \gamma \\
& =\frac{1}{2\left(2 \omega_{B D}+3\right) r}\left(\frac{\omega_{B D}+2}{12 \omega_{B D}+19}\right)^{1 / 4}\left(\frac{15 G}{2 c^{11}}\right)^{1 / 4} \frac{M_{c}^{5 / 4}}{\tau^{1 / 4}} \sin ^{2} \gamma
\end{aligned}
$$

In the last expression, we have introduced the definition of the chirp mass $M_{c}=$ $\left(m_{1} m_{2}\right)^{3 / 5} / m^{1 / 5}$. 


\section{Detectability of the scalar GWs}

Let us now study the interaction of the scalar GWs with two types of GW detectors.

As usual, we characterize the sensitivity of the detector by the spectral density of

strain $S_{h}(f)[\mathrm{Hz}]^{-1}$. The optimum performance of a detector is obtained by filtering the output with a filter matched to the signal. The energy signal-to-noise ratio $S N R$ of the filter output is given by the well-known formula:

$$
S N R=\int_{-\infty}^{+\infty} \frac{|H(f)|^{2}}{S_{h}(f)} d f
$$

where, in our case, $H(f)$ is the Fourier transform of the scalar gravitational waveform $h_{s}(t)=G \xi_{0}(t)$.

We must now take into account the astrophysical restrictions on the validity of the waveform (4.7) which is obtained in the Newtonian approximation for point-like masses. In the following, we will take the point of view that this approximation breaks down when there are five cycles remaining to collapse [24, 25].

The five-cycles limit will be used to restrict the range of $M_{c}$ over which our analysis will be performed. From (4.4), one can obtain

$$
\begin{aligned}
\omega_{g}(\tau) & =2 \omega_{0}=2 \sqrt{\frac{G m}{d^{3}}} \\
& =2\left(\frac{15 c^{5}}{64 G^{5 / 3}}\right)^{3 / 8}\left(\frac{\omega_{B D}+2}{12 \omega_{B D}+19}\right)^{3 / 8} \frac{1}{M_{c}^{5 / 8}} \tau^{3 / 8}
\end{aligned}
$$

Integrating (5.2) yields the amount of phase until coalescence

$$
\chi(\tau)=\frac{16}{5}\left(\frac{15 c^{5}}{64 G^{5 / 3}}\right)^{3 / 8}\left(\frac{\omega_{B D}+2}{12 \omega_{B D}+19}\right)^{3 / 8}\left(\frac{\tau}{M_{c}}\right)^{5 / 8}
$$

Setting (5.3) equal to the limit period, $T_{5}$ cycles $=5(2 \pi)$, solving for $\tau$ and using (5.2) leads to

$$
\omega_{5 \text { cycles }}=2 \pi(6870 \mathrm{~Hz})\left(\frac{\omega_{B D}+2}{12 \omega_{B D}+19}\right)^{3 / 5} \frac{M_{\odot}}{M_{c}}
$$


Taking $\omega_{B D}=600$, the previous limit reads

$$
\omega_{5 \text { cycles }}=2 \pi(1547 \mathrm{~Hz}) \frac{M_{\odot}}{M_{c}}
$$

\section{$5.1 \quad$ Interferometers}

An interferometric detector measures the relative displacements between the mirrored faces of test masses arranged in the L-shaped configuration of a Michelson interferometer. The directivity antenna pattern for a tensorial wave is such that the maximum detector output is obtained for a wave inpinging perpendicularly respect to the plane defined by the interferometer arms. On the contrary a scalar $\mathrm{BD}$ wave (which is also transverse) inpinging in the same direction will give a null effect. In the case of a scalar wave, the maximum effect will be obtained for a wave propagating along one interferometer arm. Assuming such a direction and setting $\sin \gamma=1$ in (4.8), we can, for instance, evaluate the $S N R$ for the VIRGO interferometer, presently under construction. We use for $S_{h}(f)$ the VIRGO noise spectrum as modeled in [19], which is the sum of three main components: thermal noise in the pendola, thermal noise in the mirrors, shot noise at high frequency:

$$
S_{h}(f)=10^{-47}\left[\alpha_{1}\left(\frac{f}{100 \mathrm{~Hz}}\right)^{-5}+\alpha_{2}\left(\frac{f}{100 \mathrm{~Hz}}\right)^{-1}+\alpha_{3}\left(\frac{f}{100 \mathrm{~Hz}}\right)^{2}\right] \mathrm{Hz}^{-1}
$$

where $\alpha_{1}=2.0, \alpha_{2}=91.8$ and $\alpha_{3}=1.23$.

Integrating (5.1) over the range $10 \mathrm{~Hz}-500 \mathrm{~Hz}$, one obtains the following $S N R$ :

$$
S N R=7.710^{4}\left(\frac{r}{\mathrm{Mpc}}\right)^{-2}\left(\frac{M_{c}}{M_{\odot}}\right)^{5 / 3}\left(\frac{\omega_{B D}+2}{12 \omega_{B D}+19}\right)^{1 / 2}\left[\frac{1}{2\left(2 \omega_{B D}+3\right)}\right]^{2}
$$

For $\omega_{B D}=600$, we find:

$$
S N R=3.810^{-3}\left(\frac{r}{\mathrm{Mpc}}\right)^{-2}\left(\frac{M_{c}}{M_{\odot}}\right)^{5 / 3}
$$

The inspiralling of two neutron stars of 1.4 solar masses each will then give $S N R=1$ at a source distance $r \simeq 70 \mathrm{kpc}$. The inspiralling of two black holes of 10 solar masses 
each will give $S N R=1$ at a distance $r \simeq 300 \mathrm{kpc}$. To get this last limit we have integrated (5.1) up to $\omega_{5}$ cycles $=2 \pi(178 \mathrm{~Hz})$.

\subsection{Spherical detectors}

A GW excites those vibrational modes of a resonant body having the proper simmetry. Most people consider the next generation of resonant-mass detectors will be of spherical shape. In the framework of the JBD theory the spheroidal modes with $l=2$ and $l=0$ are sensitive to the incoming GW [2, 20]. Thanks to its multimode nature, a single sphere is capable of detecting GW's from all directions and polarizations. We evaluate the $S N R$ of a resonant-mass detector of spherical shape for its quadrupole mode with $m=0$ and its monopole mode. In a resonant-mass detector, $S_{h}(f)$ is a resonant curve and can be characterized by its value at resonance $S_{h}\left(f_{n}\right)$ and by its half height width 21]. $S_{h}\left(f_{n}\right)$ can thus be written as

$$
S_{h}\left(f_{n}\right)=\frac{G}{c^{3}} \frac{4 k T}{\sigma_{n} Q_{n} f_{n}}
$$

Here $\sigma_{n}$ is the cross-section associated with the $n$-th resonant mode, $T$ is the thermodynamic temperature of the detector and $Q_{n}$ is the quality factor of the mode.

The half height width of $S_{h}(f)$ gives the bandwidth of the resonant mode

$$
\Delta f_{n}=\frac{f_{n}}{Q_{n}} \Gamma_{n}^{-1 / 2}
$$

Here, $\Gamma_{n}$ is the ratio of the wideband noise in the $n$-th resonance bandwidth to the narrowband noise.

From the resonant-mass detector viewpoint, the chirp signal can be treated as a transient GW, depositing energy in a time-scale short with respect to the detector 
damping time. We can then consider constant the Fourier transform of the waveform within the band of the detector and write 21]

$$
S N R=\frac{2 \pi \Delta f_{n}\left|H\left(f_{n}\right)\right|^{2}}{S_{h}\left(f_{n}\right)}
$$

The cross-sections associated to the vibrational modes with $l=0$ and $l=2$, $m=0$ are respectively 22

$$
\begin{aligned}
\sigma_{(n 0)} & =H_{n} \frac{G M v_{s}^{2}}{c^{3}\left(\omega_{B D}+2\right)} \\
\sigma_{(n 2)} & =\frac{F_{n}}{6} \frac{G M v_{s}^{2}}{c^{3}\left(\omega_{B D}+2\right)}
\end{aligned}
$$

All parameters entering the previous equation refer to the detector $M$ is its mass, $v_{s}$ the sound velocity and the constants $H_{n}$ and $F_{n}$ are given in [22]. The signal-to noise ratio can be calculated analytically by approximating the waveform with a truncated Taylor expansion around $t=0$, where $\omega_{g}(t=0)=\omega_{n l}$ [23, 24]

$$
h_{s}(t) \approx G \xi_{0}(t=0) \sin \left[\omega_{n l} t+\frac{1}{2}\left(\frac{d \omega}{d t}\right)_{t=0} t^{2}\right]
$$

Using quantum limited readout systems, one finally obtains

$$
\begin{aligned}
\left(S N R_{n}\right)_{l=0}= & \frac{5 \cdot 2^{1 / 3} H_{n} G^{5 / 3}}{32\left(\omega_{B D}+2\right)\left(12 \omega_{B D}+19\right) \hbar c^{3}} \\
\left(S N R_{n}\right)_{l=2}= & \frac{M_{c}^{5 / 3} M v_{s}^{2}}{r^{2} \omega_{n 0}{ }^{4 / 3}} \sin ^{4} \gamma \\
& \frac{5 \cdot 2^{1 / 3} F_{n} G^{5 / 3}}{} \\
& \frac{\left.M_{c}^{5 / 3} M \omega_{s D}{ }^{2}+2\right)\left(12 \omega_{B D}+19\right) \hbar c^{3}}{r^{2} \omega_{n 0}{ }^{4 / 3}} \sin ^{4} \gamma
\end{aligned}
$$

which are respectively the signal-to-noise ratio for the modes with $l=0$ and $l=2$, $m=0$ of a spherical detector.

It has been proposed to realize spherical detectors with 3 meters diameter, made of copper alloys, with mass of the order of 100 tons [26]. This proposed detector has resonant frequencies of $\omega_{12}=2 \pi \cdot 807 \mathrm{rad} / \mathrm{s}$ and $\omega_{10}=2 \pi \cdot 1655 \mathrm{rad} / \mathrm{s}$. In the 
case of optimally oriented orbits (inclination angle $\gamma=\pi / 2$ ) and $\omega_{B D}=600$, the inspiralling of two compact objects of 1.4 solar masses each will then be detected with $S N R=1$ up to a source distance $r\left(\omega_{10}\right) \simeq 30 \mathrm{kpc}$ and $r\left(\omega_{12}\right) \simeq 30 \mathrm{kpc}$.

\section{Summary and conclusions}

In this paper we have studied the waveforms emitted by a system of binary stars in the framework of the JBD theory and computed the power emitted in GW's for the tensor and scalar components. Eventually we derived limits for the detectability of such signals by interferometers and resonant mass detectors. In the former case we left aside the question of the detectability of the scalar component of the GW [3] and we have concentrated on waves impinging from the most favourable direction. We would now like to comment The detectability ranges obtained in Sections 5.1,5.2 for the scalar component of the GW's emitted by a binary system, vary from few tens to few hundreds of kpc's for masses ranging from those of typical neutron stars $\left(1.4 M_{\odot}\right)$ to those of typical black holes $\left(10 M_{\odot}\right)$. We remind the reader that for the purely tensorial component (in this case the results obtained in the framework of the JBD theory are practically the same of those of GR) the detectability range (for $1.4 M_{\odot}$ ) is $r \simeq 120 \mathrm{Mpc}$ for spherical detectors [25] and $r \simeq 300 \mathrm{Mpc}$ for interferometers [19]. The expected rate of coalescence events is of the order of 1 per year up to $100 \mathrm{Mpc}$ [27. We can thus conclude that binary systems look less promising than gravitational collapses [22] as sources of detectable scalar GW from the next generation of earth-based detectors. 


\section{A Appendix}

In order to calculate the coefficients $X_{j m}$ defined in (3.13), let us first express $X_{j m}$ as a sum of Fourier components

$$
X_{j m}(t)=\sum_{n=-\infty}^{+\infty}\left(X_{j m}\right)_{n} e^{i n \beta}
$$

where $\beta$ is the mean anomaly which, in terms of the orbital frequency $\omega_{0}$ and of the time of periastron passage $T_{0}$ (or equivalently in terms of the eccentric anomaly $\alpha$ and the eccentricity), results

$$
\beta=\omega_{0}\left(t-T_{0}\right)=\alpha-e \sin \alpha
$$

In the so-called quadrupole approximation $y=\omega d / c \ll 1$, the spherical Bessel functions $j_{l}(y)$, which appear in (3.13), can be written as

$$
j_{l}(y)=\sum_{k=0}^{\infty} \frac{(-1)^{k} y^{l+2 k}}{2^{k} k !(2 l+2 k+1) ! !}
$$

Making use of the Newtonian approximation (including only two terms of the series (A.3) ), from (2.11) and (3.13) one can obtain $X_{00}$ as

$$
X_{00}=\frac{4 \pi i \omega \varphi_{0}}{\omega_{B D}+2} \int_{V}\left(1-\frac{\left(\omega r^{\prime}\right)^{2}}{6}\right) Y_{00} \rho\left(1+\Pi-3 \frac{p}{\rho}+\frac{2 \omega_{B D}+1}{\omega_{B D}+2} U\right) d V
$$

To simplify (A.4) we use the post-Newtonian expressions of the conserved quantities 14

$$
\begin{aligned}
P^{0} & =\int \rho\left(1+v^{2}+\frac{5 \omega_{B D}+4}{2\left(\omega_{B D}+2\right)} U+\Pi\right) d \vec{x} \\
P^{i} & =\int \rho\left(1+v^{2}+\frac{5 \omega_{B D}+4}{2\left(\omega_{B D}+2\right)} U+\Pi+\frac{p}{\rho}\right) v^{i} d \vec{x} \\
& -\frac{1}{2} \int \rho\left(1+\frac{v^{2}}{2}+\frac{3\left(\omega_{B D}+1\right)}{\omega_{B D}+2} U\right) W^{i} d \vec{x}
\end{aligned}
$$

where

$$
W^{i}=\int \rho^{\prime} \frac{\left[\vec{v}^{\prime} \cdot\left|\vec{x}-\vec{x}^{\prime}\right|\right]\left(x-x^{\prime}\right)_{i}}{\left|\vec{x}-\vec{x}^{\prime}\right|^{3}} d \vec{x}^{\prime}
$$


To the required accuracy and modulo constants, one then obtains

$$
X_{00}=-\frac{\sqrt{4 \pi} i \omega \varphi_{0} \mu}{\omega_{B D}+2}\left(v^{2}+\frac{(\omega d)^{2}}{6}+\frac{m}{r}\right)
$$

In terms of the eccentric anomaly $\alpha$, the above expression reads

$$
X_{00}(t)=-\frac{\sqrt{4 \pi} i \omega \varphi_{0} \mu m}{a\left(\omega_{B D}+2\right)} G_{j=0}
$$

where

$$
G_{j=0}=\frac{1+e \cos \alpha}{1-e \cos \alpha}+\frac{n^{2}}{6}(1-e \cos \alpha)^{2}+\frac{1}{1-e \cos \alpha}
$$

Let us express $G_{j=0}$ as a sum of Fourier components

$$
G_{j=0}=\sum_{n=0}^{\infty} A_{n} \cos (n \beta)
$$

where $\beta$ is the mean anomaly defined in (A.2). Using the integral expression of the Bessel functions

$$
J_{n}(x)=\frac{1}{\pi} \int_{0}^{\pi} \cos (n \alpha-x \sin \alpha) d \alpha
$$

and the recursion formula

$$
J_{n+1}(x)+J_{n-1}(x)=\frac{2 n}{x} J_{n}(x)
$$

results

$$
A_{n}=\frac{2}{\pi} \int_{0}^{\pi} G_{j=0} \cos (n \beta) d \beta=\frac{16}{3} J_{n}(n e)
$$

Then one finally has

$$
\left(X_{00}\right)_{n}=-\frac{16 \sqrt{2 \pi}}{3} \frac{i \omega_{0} \varphi_{0}}{\omega_{B D}+2} \frac{m \mu}{a} n J_{n}(n e)
$$

Choosing the orbital plane as the $x-y$ plane, yields $X_{10}=0$ and

$$
X_{1 \pm 1}=\frac{4 \pi i \omega \varphi_{0}}{\omega_{B D}+2} \int_{V} \frac{\omega r^{\prime}}{3} Y_{1 \pm 1}^{*}(\theta, \phi) \rho\left(1+\Pi-3 \frac{p}{\rho}+\frac{2 \omega_{B D}+1}{\omega_{B D}+2} U\right)
$$

Defining $\mathcal{G}=\Omega_{2} / m_{2}-\Omega_{1} / m_{1}$, one then obtains to the required order

$$
X_{1 \pm 1}=\frac{4 \pi i \omega^{2} \varphi_{0} \mu \mathcal{G}}{\sqrt{6 \pi}\left(\omega_{B D}+2\right)} d(\cos \psi \mp i \sin \psi)
$$


where $\psi$ is the true anomaly. In terms of the eccentric anomaly $\alpha$, (A.17) results

$$
X_{1 \pm 1}=-\sqrt{\frac{2 \pi}{3}} \frac{2 i \omega^{2} \varphi_{0} \mathcal{G} \mu a}{\omega_{B D}+2} G_{j=1}
$$

where

$$
G_{j=1}= \pm(\cos \alpha-e)-i\left(1-e^{2}\right)^{1 / 2} \sin \alpha
$$

The binary system center of mass calculated with respect to the gravitational selfenergies $\Omega_{a}$ doesn't coincide with the center of mass with respect to the inertial masses $m_{a}$ of the two bodies, if the masses are different (Nordtvedt effect): the resulting dipole moment is, as we have seen, a source of scalar radiation. If we express $G_{j=1}$ as a sum of Fourier components

$$
G_{j=1}=B_{0}+\sum_{n=1}^{\infty}\left[B_{n} \cos (n \beta)+C_{n} \sin (n \beta)\right]
$$

we obtain

$$
B_{n}= \pm \frac{2}{n} J_{n}^{\prime}(n e)
$$

(where the prime indicates derivative with respect to the argument ne) and

$$
C_{n}=\frac{2}{\pi} \int_{0}^{\pi} G_{j=1} \sin (n \beta) d \beta=-\frac{2 i}{n e}\left(1-e^{2}\right)^{1 / 2} J_{n}(n e)
$$

Then the $n$-th component of the coefficient $X_{1 \pm 1}$ is

$$
\begin{aligned}
\left(X_{1 \pm 1}\right)_{n}= & -\sqrt{\frac{2 \pi}{3}} \frac{2 i \omega_{0}^{2} \varphi_{0}}{\omega_{B D}+2} \mathcal{G} \mu a \\
& {\left[ \pm J_{n}^{\prime}(n e)-\frac{1}{e}\left(1-e^{2}\right)^{1 / 2} J_{n}(n e)\right] }
\end{aligned}
$$

Finally, in the case $j=2$ one obtains $X_{2 \pm 1}=0$ and

$$
\begin{aligned}
X_{20} & =\frac{4 \pi i \omega^{3} \varphi_{0}}{15\left(\omega_{B D}+2\right)} \int_{V} r^{\prime 2} Y_{20}^{*}(\theta, \phi) \rho d V \\
X_{2 \pm 2} & =\frac{4 \pi i \omega^{3} \varphi_{0}}{15\left(\omega_{B D}+2\right)} \int_{V} r^{\prime 2} Y_{2 \pm 2}^{*}(\theta, \phi) \rho d V
\end{aligned}
$$

and in terms of $\alpha$, using the Newtonian approximation,

$$
\begin{aligned}
X_{20}(t) & =\sqrt{\frac{\pi}{5}} \frac{i \omega^{3} \varphi_{0} \mu a^{2}}{3\left(\omega_{B D}+2\right)}(1-\cos \alpha)^{2} \\
X_{2 \pm 2} & =\mp \sqrt{\frac{\pi}{30}} \frac{i \omega^{3} \varphi_{0} \mu a^{2}}{\left(\omega_{B D}+2\right)} G_{j=2}
\end{aligned}
$$


where

$$
G_{j=2}=D_{0}+\sum_{n=1}^{\infty}\left[D_{n} \cos (n \beta)+E_{n} \sin (n \beta)\right]
$$

Calculating $D_{n}$ and $E_{n}$

$$
\begin{aligned}
& D_{n}=-\frac{4}{n}\left[\frac{1}{n e^{2}}\left(2-e^{2}\right) J_{n}(n e)+\frac{2}{e}\left(e^{2}-1\right) J_{n}^{\prime}(n e)\right] \\
& E_{n}=\mp \frac{8 i}{n}\left[\frac{1-e^{2}}{e^{2}} J_{n}(n e)-\frac{1}{n e} J_{n}^{\prime}(n e)\right]
\end{aligned}
$$

the $n$-th components result

$$
\begin{aligned}
& \left(X_{20}\right)_{n}=\frac{2}{3} \sqrt{\frac{\pi}{5}} \frac{i \omega_{0}^{3} \varphi_{0}}{\omega_{B D}+2} \mu a^{2} n J_{n}(n e) \\
& \left(X_{2 \pm 2}\right)_{n}=\mp 2 \sqrt{\frac{\pi}{30}} \frac{i \omega_{0}^{3} \varphi_{0}}{\omega_{B D}+2} \mu a^{2} \\
& \frac{1}{n}\left\{\left(e^{2}-2\right) J_{n}(n e) /\left(n e^{2}\right)+2\left(1-e^{2}\right) J_{n}^{\prime}(n e) / e\right. \\
& \left.\mp 2\left(1-e^{2}\right)^{1 / 2}\left[\left(1-e^{2}\right) J_{n}(n e) / e^{2}-J_{n}^{\prime}(n e) /(n e)\right]\right\}
\end{aligned}
$$

\section{B Appendix}

We want to determine explicitly the form of the scalar GWs radiated by a binary system. From (3.12) and (A.8), (A.17), (A.24), A.25), and taking into account that in the limit $r \rightarrow \infty$ the spherical Hankel functions become

$$
h_{l}^{(1)}(\omega r) \sim \frac{e^{i[\omega r-(l+1) \pi / 2]}}{\omega r}
$$

one can easily obtain

$$
\begin{aligned}
\xi(\vec{x}, \omega)= & -\frac{2 \mu}{\left(2 \omega_{B D}+3\right) G r} e^{i \omega r} \\
& \left\{v^{2}+m / d+(\omega d)^{2} / 6+2 i \omega\left(\Omega_{2} / m_{2}-\Omega_{1} / m_{1}\right) \hat{n} \cdot \vec{d}\right. \\
& \left.-\omega^{2} d^{2}\left(3 n_{z}{ }^{2}-1\right) / 12+\omega^{2}\left[(\hat{n} \cdot \vec{d})^{2}-\left(n_{x} d_{y}-n_{y} d_{x}\right)^{2}\right] / 4\right\}_{\omega}
\end{aligned}
$$

where the subscript $\omega$ indicates that all the quantities in the right member of the above expression (B.2) are to be considered as Fourier components with frequency $\omega$ (for example, $v \rightarrow v(\omega)$ ). 
The time dependent amplitude is 14

$$
\begin{aligned}
& \xi(\vec{x}, t)=\xi(\vec{x}, \omega) e^{-i \omega t}+c . c .=-\frac{2 \mu}{\left(2 \omega_{B D}+3\right) G r} \\
& {\left[v^{2}+\frac{m}{d}-\frac{1}{6} \frac{d^{2}}{d t^{2}}\left(d_{k} d^{k}\right)\left(1-\frac{3 n_{z}^{2}-1}{2}\right)-2 \mathcal{G} \hat{n} \cdot \vec{v}-\frac{d^{2}}{d t^{2}}(\hat{n} \cdot \vec{d})^{2}-\frac{\left(n_{x} d_{y}-n_{y} d_{x}\right)^{2}}{4}\right]} \\
& =-\frac{2 \mu}{\left(2 \omega_{B D}+3\right) G r}\left[v^{2}+\frac{m}{d}-2 \mathcal{G} \hat{n} \cdot \vec{v}-(\hat{n} \cdot \vec{v})^{2}+\frac{m}{d^{3}} \hat{n} \cdot \vec{d}\right]
\end{aligned}
$$

where we have set $v=v(\omega) e^{-i \omega(t-r)}+$ c.c., ecc. 


\section{References}

[1] Gravitational Waves, Proceedings of the Second E. Amaldi Conference, E. Coccia, G. Pizzella, G. Veneziano (eds.) (World Scientific, Singapore, 1998).

[2] M. Bianchi, E. Coccia, C.N. Colacino, V. Fafone and F. Fucito, Class. and Quantum Grav. 13, 2865 (1996).

[3] M. Shibata, K. Nakao and T. Nakamura, Phys. Rev. D50, 7304 (1994).

[4] J.H. Taylor, Rev.Mod.Phys. 66, 711 (1994).

[5] C.M. Will and H.W. Zaglauer, Astrophys.J.346, 366 (1989).

[6] M.A. Scheel, S.L. Shapiro and S.A. Teukolsky, Phys. Rev. D51, 4236 (1995).

[7] T. Harada, T. Chiba, K. Nakao and T. Nakamura, Phys. Rev. D55, 2024 (1997).

[8] M. Saijo, H. Shinkai and K. Maeda, Phys. Rev. D56, 785 (1997).

[9] T. Damour and G. Esposito-Farèse, Phys. Rev. D54, 1474 (1996); grqc/9803031.

[10] T. Damour and G. Esposito-Farèse, gr-qc/9803031.

[11] J. Novak, Phys. Rev. D57, 4789 (1998).

[12] P. Jordan Z. Phys. 157, 112 (1959); C. Brans and R.H. Dicke, Phys. Rev. 124 925 (1961).

[13] J. Miller, M. Schneider, M. Soffel and H. Ruder, Astrophys. J. Lett. 382, L101 (1991).

[14] C.M. Will, Theory and Experiment in Gravitational Physics (Cambridge University Press, Cambridge, 1993).

[15] D.L. Lee, Phys. Rev D10, 2374 (1974). 
[16] P.C. Peters and J. Mathews, Phys. Rev 131, 435 (1963).

[17] J. Mathews, J. Soc. Indust. Appl. Math. 10, 768 (1962).

[18] L. Blanchet and G. Schäfer, Mon. Not. R. astr. Soc. 239, 845 (1989).

[19] M. Beccaria, G. Cella, A. Ciampa, E. Cuoco, G. Curci and A. Vicere', Virgo note NTS96-24 (1996).

[20] J.A. Lobo, Phys.Rev. D52, 591 (1995).

[21] P. Astone, G.V. Pallottino and G. Pizzella, Class. Quantum Grav. 14, 2019 (1997).

[22] M. Bianchi, M. Brunetti, E. Coccia, F. Fucito and J. A. Lobo, gr-qc/9709045, to appear in Phys. Rev. D.

[23] J. P. A. Clark and D. M. Eardley, Astrophys. J. 215, 311 (1977).

[24] D. Dewey, Phys. Rev. D36, 1577 (1987).

[25] E. Coccia and V. Fafone, Phys. Letters A213, 16 (1996).

[26] G. Frossati and E. Coccia, Cryogenics 34 (ICEC Supplement), 9 (1994). 304 (1994).

[27] E.S. Phinney, Astrophys. J. 380, L17 (1991). 